\title{
COPREVALÊNCIA DE HANSENÍASE EM CONTATOS COM IDADE ENTRE 5 E 15 ANOS NO NORDESTE BRASILEIRO
}

\section{HANSENIASIS COPREVALENCE IN CONTACTS WITH AGE BETWEEN 5 AND 15 YEARS IN THE NORTHEAST OF BRAZIL}

\section{COPREVALENCIA DE HANSENIASIS EN CONTACTOS CON EDAD ENTRE 5 Y 15 AÑOS EN EL NORDESTE BRASILEÑO}

\author{
Fernanda da Silva Goes ${ }^{1}$ \\ Clodis Maria Tavares ${ }^{2}$ \\ Jovânia Marques de Oliveira e Silva ${ }^{3}$ \\ Tâmyssa Simões dos Santos ${ }^{4}$ \\ Nataly Mayara Cavalcante Gomes \\ Karen da Silva Santos ${ }^{6}$
}

Como citar este artigo: Goes FS, Tavares CM, Silva JMO, Santos TS, Gomes NMC, Santos KS. Coprevalência de hanseníase em contatos com idade compreendida entre 5 a 15 anos no Nordeste brasileiro. Rev baiana enferm. 2018;32:e26100.

Objetivo: avaliar a coprevalência da hanseníase em contatos com idade compreendida entre 5 e 15 anos. Método: estudo descritivo, com abordagem quantitativa, realizado no período de novembro de 2014 a julho de 2015 . A população do estudo foi composta por contatos intradomiciliares e peridomiciliares de pessoas atingidas pela hanseníase, que estivessem na faixa etária de 5 a 15 anos. A amostra estudada foi constituída de 73 contatos. Resultados: do total de avaliados, 30 (41,0\%) foram intradomiciliares e 43 (59,0\%), peridomiciliares. Entre os contatos, 27 (37,0\%) foram considerados sintomáticos dermatológicos; destes, 17 (23,3\%) suspeitos para hanseníase por apresentarem de uma a oito lesões, sendo a maioria mancha hipocrômica (82,3\%). Conclusão: entre os contatos examinados neste estudo, com idades compreendidas entre 5 e 15 anos, a coprevalência da hanseníase não foi diagnosticada, mas o número de contatos classificados como suspeitos foi elevado, o que se torna preocupante do ponto de vista epidemiológico.

Descritores: Hanseníase. Enfermagem. Busca de comunicante. Anamnese. Exame físico.

Objective: to evaluate the co-prevalence of leprosy in contacts between the ages of 5 and 15 years. Method: this is a descriptive study, with a quantitative approach, carried out from November 2014 to July 2015. The study population was composed of intradomiciliary and peridomiciliary contacts of people affected by leprosy who were in the age

\footnotetext{
Enfermeira. Porto de Pedras, Alagoas, Brasil.

Enfermeira. Doutora em Ciências. Pesquisadora do Conselho Nacional de Desenvolvimento Científico e Tecnológico. Professora Adjunta da Universidade Federal de Alagoas. Maceió, Alagoas, Brasil.

Enfermeira. Doutora em Enfermagem. Professora Adjunta da Universidade Federal de Alagoas. Maceió, Alagoas, Brasil.

Enfermeira. Mestre em Educação em Ciências da Saúde. Docente do Centro Universitário Mauricio de Nassau. Maceió, Alagoas, Brasil. simoestamyssa@gmail.com

Estudante de Enfermagem, Escola de Enfermagem e Farmácia da Universidade Federal de Alagoas. Maceió, Alagoas, Brasil.

Enfermeira. Mestre em Ciências. Chefe de Seção de Vigilância Epidemiológica do município de Sertãozinho. Sertãozinho, São Paulo, Brasil.
} 
range of 5 to 15 years. The sample consisted of 73 contacts. Results: of the total of evaluated, 30 (41.0\%) were intradomiciliary and 43 (59.0\%), peridomiciliary. Among the contacts, 27 (37.0\%) were considered dermatological symptomatic; Of these, 17 (23.3\%) suspected leprosy patients had one to eight lesions, most of them hypochromic spots (82,3\%). Conclusion: among the contacts examined in this study, aged between 5 and 15 years, the co-prevalence of leprosy was not diagnosed, but the number of contacts classified as suspects was high, which is worrisome from the epidemiological point of view.

Descriptors: Leprosy. Nursing. Search for communicator. Anamnesis. Physical exam.

Objetivo: evaluar la coprevalencia de la lepra en contactos con edad comprendida entre 5 y 15 años. Método: estudio descriptivo, con abordaje cuantitativo, de noviembre de 2014 a julio de 2015. Población del estudio compuesta por contactos intradomiciliares y peridomiciliares de personas afectadas por la lepra, que estuvieran en el grupo de edad de 5 a 15 años. Muestra estudiada de 73 contactos. Resultados: del total de evaluados, 30 (41,0\%) fueron intradomiciliares y 43 (59,0\%), peridomiciliares. Entre los contactos, 27 (37,0\%) fueron considerados sintomáticos dermatológicos; de estos, 17 (23,3\%) sospechosos de lepra por presentar de una a ocho lesiones, siendo la mayoría mancha hipocrómica (82,3\%). Conclusión: entre los contactos examinados en este estudio, con edades entre 5 y 15 años, la coprevalencia de la lepra no fue diagnosticada, pero el número de contactos clasificados como sospechosos fue elevado, lo que se vuelve preocupante desde el punto de vista epidemiológico.

Descriptores: Lepra. Enfermería. Trazado de contacto. Anamnesis. Examen físico.

\section{Introdução}

A hanseníase é uma doença infecciosa milenar de evolução crônica, com amplo espectro de manifestações clínicas e dermatoneurológicas e tem como agente etiológico o Mycobacterium leprae. Este também é conhecido como Bacilo de Hansen, bactéria que possui tropismo por pele e nervos periféricos. Quando as pessoas são atingidas por esta doença e não são diagnosticadas precocemente, tendem a evoluir com deformidades e incapacidades físicas, o que potencializa o estigma social e o preconceito decorrentes dessa doença ${ }^{(1-2)}$.

A transmissão da doença ocorre por meio das vias aéreas superiores, sendo necessário um contato prolongado com uma pessoa atingida pela hanseníase que apresenta a forma multibacilar sem tratamento. A coprevalência da doença representa o risco aumentado em até 10 vezes de haver mais de um caso no mesmo domicílio. A cadeia de transmissão pode ser interrompida pelo diagnóstico precoce entre os contatos intradomiciliares e peridomiciliares, além de tratamento da fonte de infecção ${ }^{(3)}$.

\begin{abstract}
De acordo com relatórios oficiais recebidos de 138 países de todas as regiões da OMS [Organização Mundial da Saúde], a prevalência registrada global de lepra no final de 2015 era de 176.176 casos $(0,2$ casos por 10.000 pessoas. O número de novos casos reportados globalmente em 2015 foi de 211.973 (2,9 novos casos por 100.000 pessoas). Em 2014, foram notificados 213.899 novos casos e, em 2013, 215.656 novos casos ${ }^{\text {(4:1, tradução nossa) }}$ ?
\end{abstract}

No mundo, em 2014, foram diagnosticados 213.875 casos novos de hanseníase, sendo 125.785 na Índia e 31.064 no Brasil. Este país encontra-se na segunda posição com relação ao número absoluto de casos de hanseníase; em 1ํ lugar, está a Índia, considerada o berço da doença $^{(5)}$. Quanto aos países da América do Sul, é conhecida em todos, com exceção do Chile continental. Relatos antigos, do século XVI, informam que a doença foi descoberta pela primeira vez na Colômbia, local que abriu o primeiro leprosário no ano de 1530, em Cartagena, cidade localizada na Costa atlântica desse país ${ }^{(6)}$.

Nesse cenário, no ano de 2015, no Brasil, o coeficiente de detecção de casos novos segundo a região foi 14,07/100 mil habitantes, correspondendo a 35.131 casos novos da doença no país.

\footnotetext{
According to official reports received from 138 countries from all WHO [World Health Organization] regions, the global registered prevalence of leprosy at the end of 2015 was 176176 cases (0.2 cases per 10000 people [1]). The number of new cases reported globally in 2015 was 211973 (2.9 new cases per 100000 people). In 2014 , 213899 new cases were reported, and in 2013,215 656 new cases.
} 
Na população menor de 15 anos, houve registro de 2.113 casos novos, com coeficiente de detecção 4,46/100.000 habitantes ${ }^{(7)}$.

Em Alagoas, os dados epidemiológicos apontam uma gradativa diminuição do coeficiente de detecção de casos de hanseníase, porém ainda exibiu alta taxa em 2015, quando foi registrado um coeficiente de detecção de 10,51/100.000 habitantes. Ao tratar desse coeficiente em menores de 15 anos em Alagoas, no ano de 2015, o coeficiente registrado foi de 2,66/100.000 habitantes, taxa considerada média segundo padrões do Ministério da Saúde (MS). Nesse ano, apenas 69,59\% dos contatos intradomiciliares foram examinados, o que ainda está abaixo da média do país $(76,63 \%)^{(7)}$.

Dados do ano de 2015 revelam que 14 casos novos no Município de Rio Largo, com um coeficiente de detecção de 19,83/100.000 habitantes ${ }^{(8)}$. Dados epidemiológicos deste município esclarecem que $62,3 \%$ dos contatos intradomiciliares de pessoas atingidas pela hanseníase foram examinados em $2013^{(9)}$.

Apesar da introdução da poliquimioterapia (PQT) no tratamento da hanseníase, ainda há desafios técnicos e operacionais a serem superados, visando sua eliminação, como, por exemplo, as baixas taxas de exames dos contatos e o diagnóstico tardio da doença ${ }^{(10)}$. Os contatos são aqueles da mesma família ou não familiares que residem no domicílio (intradomiciliares) ou que moram nas proximidades da residência da pessoa que tem hanseníase (peridomiciliares), no período mínimo de três meses até cinco anos antes do diagnóstico $^{(11)}$.

Nesta perspectiva, a detecção de casos de hanseníase em menores de 15 anos gera um sinal de alarme, pois representa hiperendemicidade na comunidade ou região, visto que indivíduos nessa faixa etária têm contato precocemente e maior proximidade física com o caso índice, indicando que há transmissão ativa e recente de pacientes multibacilares, e estes não foram devidamente diagnosticados e tratados ${ }^{(2-3)}$. O risco de desenvolvimento da doença inerente a essa faixa etária ainda é maior devido à dificuldade para a realização do diagnóstico precoce, o que confere maior probabilidade de evolução para complicações e deformidades ${ }^{(2)}$.

A sistematização do cuidado de enfermagem, conforme estabelecido no Programa de Eliminação de Hanseníase, compreende a suspeição da doença em diagnóstico precoce, resultante de exames dermatoneurológicos, prevenção de incapacidades, apoio emocional, acompanhamento do tratamento e consequente cura da doença $^{(12)}$.

Dessa forma, este estudo tem como objetivo avaliar a coprevalência da hanseníase em contatos com idade compreendida entre 5 e 15 anos.

\section{Método}

Trata-se de um estudo descritivo, com abordagem quantitativa, realizado no Município de Rio Largo, Alagoas, Brasil.

A população do estudo foi composta por 148 contatos intradomiciliares, de 52 casos índices multibacilar, e 43 peridomiciliar, considerados os indivíduos que convivem no raio de 50 metros de um paciente diagnosticado na forma multibacilar. Este dado foi elucidado em estudo ${ }^{(11)}$ realizado no estado de Mato-Grosso, que observou a ocorrência de 35,3\% dos casos novos de hanseníase entre indivíduos que residiam próximos de pessoas atingidas por essa doença.

Os critérios de inclusão foram: contatos intra e peridomiciliares com idade compreendida entre 5 e 15 anos que residiam no endereço cadastrado. A escolha da faixa etária a partir de 5 anos de idade para a realização do estudo justifica-se devido ao longo período de incubação do bacilo ${ }^{(1)}$, o que representa, na maioria dos casos, a manifestação dos sintomas tardiamente. Além disso, a avaliação dos contatos foi realizada por intermédio do exame dermatoneurológico; portanto, crianças a partir de 5 anos já conseguem responder ao exame com maior concentração, o que facilita a aplicação do teste de sensibilidade $^{(2)}$.

Não foram estabelecidos critérios de exclusão. Contudo, a população final do estudo foi composta por 73 contatos, sendo 30 intradomiciliares e 43 peridomiciliares, pois não foram estudados 
os contatos que não estavam nas residências durante a visita domiciliar e aqueles que os pais não autorizaram a participação na pesquisa.

A coleta de dados ocorreu no período de novembro de 2014 a julho de 2015. Na primeira etapa, foi realizado o levantamento do quantitativo de casos índices de hanseníase (forma multibacilar), no período de 2009 a 2014, e seus respectivos contatos no Sistema de Informação de Agravos de Notificação (SINAN) e no Boletim Epidemiológico do Estado. Na segunda etapa, realizaram-se visitas às Unidades Básicas de Saúde (UBS), nas quais os pacientes diagnosticados com hanseníase foram cadastrados. Ressalta-se que as informações sobre os contatos intradomiciliares nos registros do Boletim Epidemiológico e no SINAN não contemplavam dados relativos às idades, o que exigiu a consulta aos prontuários para a coleta dessas informações. $\mathrm{Na}$ terceira etapa, foram descritos os endereços dos casos índice, no intuito de realizar as visitas domiciliares. Estas foram executadas com a presença dos Agentes Comunitários de Saúde (ACS). Torna-se relevante mencionar que, nessa ocasião, os contatos peridomiciliares foram identificados e incluídos na pesquisa. Durante as visitas, os pais/responsáveis dos contatos foram convidados e orientados sobre a pesquisa. Após o aceite, solicitou-se a assinatura de todos no Termo de Consentimento Livre e Esclarecido (TCLE) e no Termo de Assentimento, que foi direcionado para os contatos que tinham idade compreendida entre 5 e 15 anos. Nesse momento foram agendadas as consultas, que seriam realizadas nas UBS.

A consulta de enfermagem dos contatos foi realizada por enfermeiras voluntárias e por alunos de graduação em Enfermagem devidamente capacitados e supervisionados pelos coordenadores da pesquisa. Constituiu-se por anamnese, exame físico geral, exame dermatoneurológico, avaliação neurofuncional e administração da vacina BCG, para os casos em que houve necessidade, conforme os critérios do MS. O objetivo dos pesquisadores, durante os exames, era realizar a suspeição de casos.
Os contatos considerados suspeitos após a consulta foram reexaminados, com o propósito de diagnosticar aqueles que se enquadravam na definição de caso de hanseníase proposta pelo MS. Em caso de dúvidas quanto ao fechamento, os casos seriam encaminhados à Unidade de Referência de Maceió para resolução, com a Ficha do "Protocolo Complementar de Investigação Diagnóstica de casos de hanseníase em menores de 15 anos" devidamente preenchida.

Para a coleta de dados foram utilizados dois instrumentos: o inquérito com informações sociodemográficas e clínicas sobre o caso índice obtidas no Sistema de Vigilância da Secretaria Municipal de Saúde de Rio Largo (AL) foi o primeiro; e o segundo continha dados referentes à parte clínica do exame de contatos intra e peridomiciliares avaliados durante a consulta de enfermagem. A análise dos dados foi realizada com a utilização do programa Epilnfo e os resultados estão apresentados em tabelas.

A todos os participantes da pesquisa foi garantido o sigilo dos dados. O presente estudo foi apreciado e aprovado pelo Comité de Ética e Pesquisa (CEP) da Universidade Federal do Ceará (UFC), com Parecer Consubstanciado n. 532.850 .

\section{Resultados}

No período de 2009 a 2014, foram registrados, no SINAN, 52 casos de hanseníase no município de Rio Largo ( $\mathrm{AL}$ ). Destes foi selecionada uma amostra de 73 contatos com idades compreendidas entre 5 e 15 anos, sendo 30 (41,0\%) intradomiciliares e $43(59,0 \%)$ peridomiciliares. Dos 30 contatos intradomiciliares identificados, apenas 16 (52,0\%) foram submetidos ao exame de contatos nas UBS, durante o ano de diagnóstico do caso índice. Quanto aos contatos peridomiciliares, não há registro no SINAN e/ou Boletim Epidemiológico, porque não são prioridades do MS.

Dos 73 contatos participantes da pesquisa, 27 (37,0\%) foram considerados sintomáticos dermatológicos para hanseníase. Destes, 20 (27,4\%) apresentaram de 1 a 5 lesões de pele, sendo a 
maioria do aspecto mancha hipocrômica $(30,1 \%)$.

Durante a avaliação neurofuncional simplificada, observou-se que $1(1,4 \%)$ contato apresentou espessamento do nervo ulnar (Tabela 1).

Tabela 1 - Distribuição dos contatos, na faixa etária de 5 a 15 anos, examinados segundo os aspectos dermatoneurológicos. Rio Largo, Alagoas, Brasil - 2015 (n=73)

\begin{tabular}{|c|c|c|}
\hline Variáveis & $\mathbf{n}$ & $\%$ \\
\hline \multicolumn{3}{|l|}{ Sintomáticos dermatológicos } \\
\hline Não & 46 & 63,0 \\
\hline Sim & 27 & 37,0 \\
\hline \multicolumn{3}{|l|}{ Quantidade de lesões dermatológicas } \\
\hline 0 & 46 & 63,1 \\
\hline 1 a 5 & 20 & 27,4 \\
\hline 6 a 8 & 7 & 9,5 \\
\hline \multicolumn{3}{|l|}{ Aspecto das lesões } \\
\hline Mancha hipercrômica & 1 & 1,4 \\
\hline Mancha hipocrômica & 22 & 30,1 \\
\hline Mancha hipocrômica e Mancha hipercrômica & 3 & 4,1 \\
\hline Mancha hipocrômica e Placa hipercrômica & 1 & 1,4 \\
\hline \multicolumn{3}{|l|}{ Nervos afetados } \\
\hline Não & 72 & 98,6 \\
\hline Sim & 1 & 1,4 \\
\hline
\end{tabular}

Fonte: Elaboração própria.

Dos 27 contatos considerados sintomáticos dermatológicos, 17 apresentaram alteração da sensibilidade térmica (hiperestesia ou hipoestesia), portanto considerados suspeitos (23,3\%), segundo a definição de caso estabelecida pela OMS e pelo MS. Destes contatos suspeitos, 2 foram confirmados com o diagnóstico de hanseníase.

Ainda no tocante a esses 17 contatos, 8 $(47,0 \%)$ eram intradomiciliares e 9 (53,0\%) peridomiciliares. Entre os casos índice desses contatos, 11 (64,7\%) foram classificados como multibacilares e 6 (35,3\%) paucibacilares. Há uma predominância, entre os contatos suspeitos da faixa etária de 5 a 8 anos $(47,06 \%)$, de indivíduos do sexo masculino (76,4\%).

Conforme observado na Tabela 2, 11 (64,7\%) contatos suspeitos possuíam uma renda familiar de aproximadamente 1 a 2 salários mínimos e 6 (35,3\%) sobreviviam com menos de 1 salário.

Tabela 2 - Distribuição dos contatos suspeitos de hanseníase com faixa etária de 5 a 15 anos, segundo características socioeconômicos. Rio Largo, Alagoas, Brasil - 2015 (n=17)

(continua)

\begin{tabular}{lcc}
\hline Variáveis & n & $\mathbf{\%}$ \\
\hline Renda familiar & & 64,7 \\
De 1 a 2 salários & 11 & 35,3 \\
Menos que 1 Salário Mínimo & 6 & \\
Residência & & 17,6 \\
$\quad$ Alugado & 3 & 82,3 \\
Casa própria & 14 & \\
Água encanada & & 29,4 \\
Não & 5 & 70,5 \\
Sim & 12 &
\end{tabular}


Tabela 2 - Distribuição dos contatos suspeitos de hanseníase com faixa etária de 5 a 15 anos, segundo características socioeconômicos. Rio Largo, Alagoas, Brasil - 2015 (n=17)

\begin{tabular}{|c|c|c|}
\hline Variáveis & $\mathbf{n}$ & $\%$ \\
\hline \multicolumn{3}{|c|}{ Saneamento básico } \\
\hline Céu aberto & 13 & 76,4 \\
\hline Fossa & 3 & 17,6 \\
\hline Rede pública & 1 & 5,8 \\
\hline \multicolumn{3}{|c|}{ Número de cômodos } \\
\hline 2 a 3 & 2 & 11,7 \\
\hline 4 a 5 & 8 & 47,0 \\
\hline 6 a 8 & 2 & 11,7 \\
\hline Não respondeu & 5 & 29,4 \\
\hline
\end{tabular}

Fonte: Elaboração própria.

Observa-se, com relação à espacionalização geográfica, que a maioria dos contatos suspeitos morava em casa própria $(82,3 \%)$, com 4 a 5 cômodos (47,0\%), água encanada (70,5\%) e eliminavam dejetos a céu aberto (76,4\%). Com relação à proximidade dos contatos suspeitos com o caso índice, 8 (47,0\%) são vizinhos. Entre os intradomiciliares, $5(29,0 \%)$ são filhos e 2 (12,0\%) sobrinhos.
A Tabela 3 mostra que, durante a realização do exame dermatoneurológico na consulta de enfermagem, os contatos suspeitos apresentaram entre 1 a 8 lesões sugestivas, sendo a maioria do tipo mancha hipocrômica (82,3\%). Destaca-se ainda que os $17(100,0 \%)$ contatos suspeitos apresentavam cicatriz da BCG.

Tabela 3 - Distribuição dos contatos suspeitos de hanseníase na faixa etária de 5 a 15 anos, segundo os aspectos clínicos da hanseníase. Rio Largo, Alagoas, Brasil - 2015 (n=17)

\begin{tabular}{l|cc}
\hline Variables & $\mathbf{n}$ & $\mathbf{\%}$ \\
\hline Quantidade de lesões sugestivas & 3 & 17,6 \\
1 & 2 & 11,7 \\
2 & 1 & 5,8 \\
3 & 3 & 17,6 \\
4 & 3 & 17,6 \\
5 & 2 & 11,7 \\
7 & 3 & 17,6 \\
8 & & 82,3 \\
Aspecto das lesões & 14 & 11,7 \\
Mancha hipocrômica & 2 & 5,9 \\
Mancha hipocrômica e Mancha hipercrômica & 1 & 94,1 \\
Mancha hipocrômica e Placa hipercrômica & & 5,8 \\
Nervos afetados & 16 & - \\
Não & 1 & 100,0 \\
Sim & & 88,2 \\
Cicatriz da BCG & - & 11,7 \\
Não & 17 & \\
Sim & 15 & \\
Número de cicatrizes da BCG & 2 & \\
1 & & \\
2 & & \\
\hline
\end{tabular}

Fonte: Elaboração própria.

Nota: Sinal convencional utilizado:

- Dado numérico igual a zero não resultante de arredondamento. 


\section{Discussão}

Com o desenvolvimento deste estudo, pôde-se identificar que a minoria dos contatos de pessoas com hanseníase foi avaliada. Dos 30 contatos intradomiciliares encontrados, apenas 17 foram submetidos à realização do exame de contatos na UBS, após o diagnóstico do caso índice. Desse modo, observa-se um número abaixo da média do Brasil (77,0\%) e de Alagoas (72,0\%) nos anos de 2012 a $2016^{(13)}$.

Em um estudo de caráter transversal e descritivo realizado no município de Cacoal (RO) apontou-se que 58,3\% dos contatos não foram orientados para a realização do exame dermatoneurológico, dado importante, uma vez que essa avaliação é indispensável ${ }^{(14)}$. Este fato reflete a precariedade das ações de vigilância em saúde, visto que esta é uma das prioridades para a eliminação da hanseníase ${ }^{(15)}$.

A vigilância dos comunicantes é um dos pilares essenciais ao controle da hanseníase. A estratégia de avaliar a co-prevalência de hanseníase em contatos intra e peridomicilares surge como uma ação fundamental que necessita ser implantada e incorporada pelos profissionais de saúde, com o objetivo de detectar de forma precoce a doença, principalmente quando se trata de crianças e pré-adolescentes ${ }^{(14)}$.

Crianças e adolescentes acometidos pela hanseníase poderão sofrer sérios impactos de ordem social, física e emocional em suas vidas, tendo em vista a probabilidade de acontecerem deformidades e incapacidades. A presença da doença na população infantil levará a alterações nas atividades domésticas e escolares, bem como nas relações de amizade ${ }^{(16)}$.

Deve-se levar em conta que a realização do exame em crianças e adolescentes é fundamental, mas exige conhecimento científico dos profissionais, para entender as respostas dos testes de sensibilidade, exames considerados criteriosos e $\operatorname{minuciosos}^{(17)}$.

Observou-se, nesta pesquisa, que a maioria dos avaliados era pré-adolescente, entre 9 e 12 anos, capazes de responder ao exame dermatoneurológico com maior concentração, o que facilitou a aplicação do teste de sensibilidade. No entanto, ainda assim, esse maior número de crianças e adolescentes examinados pode ser visto como baixo.

A não adesão ao exame pode estar atrelada a fatores como ausência do agendamento e de informação sobre a sua importância, distância da residência do contato até uma UBS, dificuldade de transporte e de encontrar um horário compatível com o trabalho e o funcionamento da UBS, entre outros ${ }^{(14)}$.

Entre as crianças e adolescentes avaliados, foram identificadas cerca de 1 a 8 lesões sugestivas, que possuíam, na sua maioria, manchas hipocrômicas, semelhante ao encontrado em outros estudos, que identificaram crianças na faixa etária em torno de 2 a 9 anos apresentando as formas paucibacilares, e as formas multibacilares na faixa etária de 10 a 14 anos. Esses achados devem-se ao maior período de incubação da doença $^{(10,17-19)}$.

Em relação ao gênero, a maioria dos contatos era do sexo masculino $(56,1 \%)$, o que se assemelha ao estudo realizado em $\mathrm{Cuba}^{(7)}$, que identificou a maior presença de meninos. Em contrapartida, um estudo retrospectivo de série de casos, na descrição do perfil epidemiológico da hanseníase em menores de 15 anos de idade em um centro de referência na região Sudeste do Brasil, encontrou predominância do sexo feminino $(56,0 \%)^{(20)}$. Apesar das distinções entre os países da América Central e do Sul, quando são avaliados os países latino-americanos, não se encontram diferenças significativas ${ }^{(6)}$.

Quanto aos dados referentes às características socioeconômicas, estão em consonância com a literatura, que relaciona as condições epidemiológicas da hanseníase e consequentemente dos seus contatos com as condições ligadas à pobreza $^{(21)}$. Além disso, vale salientar que a zona de residência predominante da maioria dos contatos foi a urbana, evidenciando a hanseníase como doença urbanizada, tendo em vista o fato de estes residirem ou serem vizinhos dos casos indice $^{(19)}$.

No tocante à vacinação, o MS determina a aplicação da vacina BCG-ID somente em contatos 
intradomiciliares no momento da avaliação. Vale salientar que os contatos peridomiciliares ainda não foram preconizados nas diretrizes do $\mathrm{MS}^{(10)}$. Todavia, neste estudo, pôde ser observada a coprevalência entre caso índice e vizinhos.

O número de contatos intradomiciliares suspeitos, com duas cicatrizes de BCG-ID, pode ser considerado abaixo do esperado (apenas um), tendo em vista que essa vacina tem sua administração recomendada ao nascer. Considerando que todos, ou pelo menos a maioria, foram vacinados após o nascimento, esperava-se que tivessem duas cicatrizes, visto que os casos de ausência teriam que ser encaminhados à vacinação no exame de contato. Em consonância, pesquisa realizada na Colômbia ${ }^{(16)}$ também encontrou baixa adesão à vacina BCG-ID em escolares.

Com relação à classificação operacional do caso índice, 11 (64,7\%) eram multibacilares. Os contatos de doentes multibacilares, devido às características do agente etiológico e consequentemente ao longo período de incubação, devem ser acompanhados por cinco anos ${ }^{(22)}$. Isto é, o exame clínico dos comunicantes como conduta epidemiológica deve ser sempre realizado, principalmente em crianças e jovens menores de 15 anos, considerados indicadores de acompanhamento da endemia ${ }^{(22)}$.

Um doente multibacilar não tratado, ou tratado de maneira inadequada, produz cerca de cinco novos doentes por ano. Por isso, a busca e o acompanhamento sistematizados dos contatos intradomiciliares é um método eficaz para o diagnóstico precoce da hanseníase, principalmente em menores de 15 anos, visto que há maior possibilidade de detectar a fonte de contágio, já que esta pode estar mais próxima física e temporalmente $\mathrm{e}^{(2,14)}$.

Quando se analisou a avaliação neurofuncional simplificada, identificou-se um contato suspeito 1 (5,8\%) apresentando espessamento do nervo ulnar. A predileção do Bacilo de Hansen por nervos periféricos faz com que a hanseníase seja caracterizada pelo aparecimento de incapacidades físicas. Por isso, para preveni-las, é necessário o diagnóstico precoce ${ }^{(2)}$.
Durante o exame dos contatos foram encontrados suspeitos para hanseníase. Estes foram encaminhados para um Centro de Referência, com o intuito de fechar ou descartar o diagnóstico. Além disso, na impossibilidade de concluir/ fechar o caso, essas crianças precisariam ser acompanhadas de forma mais sistematizada por parte da equipe de saúde. A maior parte dos suspeitos era do sexo masculino. Segundo a OMS, os homens são afetados pela hanseníase com mais frequência do que as mulheres, geralmente na proporção de 2:1, em grande parte do mundo. Essa preponderância de homens é observada em diversas situações geográficas, como Índia, Filipinas, Havaí, Venezuela e Camarões ${ }^{(6)}$.

Houve também predominância, entre os contatos suspeitos, da faixa etária de 5 a 8 anos $(47,0 \%)$, o que diverge de outro estudo ${ }^{(23)}$, no qual a maioria das crianças foi diagnosticada entre 10 e 14 anos. O público desse estudo é semelhante ao de outra pesquisa ${ }^{(21)}$ sobre hanseníase no Brasil, na qual é composto de pessoas com baixa renda e concentrado em bairros menos privilegiados, mais uma vez comprovando a persistente exclusão social que acompanha os portadores de hanseníase e consequentemente seus contatos.

Uma das limitações deste estudo foi a redução da amostra em decorrência das dificuldades de realização da pesquisa, motivada por mudanças de endereço dos contatos, duplicação de dados no SINAN, prontuários incompletos, dificuldade de agendamento de horários para as consultas de enfermagem. Neste caso, por se tratar de menores de idade, que só poderiam ir às consultas acompanhados por seus pais e/ou responsáveis, e estes, em virtude das duplas jornadas de trabalho e dos horários de aulas das crianças e dos adolescentes, não podiam aprazar as consultas.

\section{Conclusão}

Este estudo permitiu concluir-se que, apesar de não ter sido realizado nenhum diagnóstico de hanseníase entre os contatos examinados, com 
idade compreendida entre 5 e 15 anos, o alto número de suspeitos $(37,0 \%)$, considerando o pequeno tamanho amostral, torna-se preocupante do ponto de vista epidemiológico. Assim, visto que aqueles que realizaram exame de contatos na unidade de saúde não foram considerados suspeitos no momento do diagnóstico do caso índice, a probabilidade de concretizar o diagnóstico é menor, se não há suspeição.

Constatou-se, neste estudo, que as medidas que visam detectar a doença de forma precoce e evitar as consequências do diagnóstico tardio e dos estigmas sociais, a exemplo do monitoramento de áreas endêmicas, acompanhamento sistemático dos contatos intradomiciliares e peridomiciliares, não são adotadas sistematicamente.

Os responsáveis por este estudo reconhecem que o adequado direcionamento do Programa da Hanseníase está intrinsicamente relacionado ao compromisso de governantes, gestores, profissionais e sociedade para a garantia do acesso da população aos serviços de saúde, com o intuito de possibilitar a prevenção, o diagnóstico precoce e o tratamento adequado para a doença.

Nesse sentido, fica evidente a importância da atuação da enfermeira para o controle da hanseníase, pois, principalmente no âmbito da atenção básica, realiza a consulta de enfermagem tanto ao portador e ex-portador da doença como aos seus contatos, com o exame dermatoneurológico, avaliação neurofuncional e acompanhamento desses durante os anos, com o intuito de realizar a suspeição da doença e possibilitar o diagnóstico precoce e situações de coprevalência.

É necessário enfatizar a importância da prática na atenção e o estudo da hanseníase nas graduações, em especial na enfermagem e na medicina, profissões que lidam com a temática com maior proximidade.

Observa-se, assim, a necessidade de estudos mais aprofundados, especialmente relacionados aos contatos nessa faixa etária ( 5 a 15 anos) que, ao serem diagnosticados, apresentam-se como importantes marcadores de transmissão da hanseníase. Ainda que a hanseníase apresente baixa letalidade e mortalidade, o acometimento de crianças, quando não diagnosticado a tempo e tratado, pode afetar o futuro dessas pessoas em decorrência dos problemas físicos, sociais e psicológicos provocados pela doença.

\section{Fonte de Financiamento:}

Este projeto teve apoio financeiro do Conselho Nacional de Desenvolvimento Científico e Tecnológico (CNPq).

\section{Colaborações:}

1. concepção, projeto, análise e interpretação dos dados: Fernanda da Silva Goes, Clodis Maria Tavares e Jovânia Marques de Oliveira e Silva;

2. redação do artigo e revisão crítica relevante do conteúdo intelectual: Fernanda da Silva Goes, Tâmyssa Simões dos Santos, Nataly Mayara Cavalcante Gomes e Karen da Silva Santos;

3. aprovação final da versão a ser publicada: Fernanda da Silva Goes, Clodis Maria Tavares, Jovânia Marques de Oliveira e Silva, Tâmyssa Simões dos Santos, Nataly Mayara Cavalcante Gomes e Karen da Silva Santos.

\section{Referências}

1. Souza MF, Vanderlei LCM, Frias PG. Avaliação da implantação do Programa de Controle da Hanseníase em Camaragibe, Pernambuco. Epidemiol Serv Saúde [Internet]. 2017 [cited 2018 Sep 4];26(4):817-34. Available from: http://dx.doi. org/10.5123/s1679-49742017000400013

2. Schneider PB, Freitas BHBM. Tendência da hanseníase em menores de 15 anos no Brasil, 2001-2016. Cad Saúde Pública [Internet]. 2018 [cited 2018 Sep 4];34(3):e00101817. Available from: http://dx.doi.org/10.1590/0102-311x00101817

3. Souza VFM, Silva RS, Valle CLP, Obadia DL, Daxbacher ELR. Report of three new leprosy cases in children under fifteen in the municipality of Itaguai, Rio de Janeiro: event alert for epidemiological investigation. An Bras Dermatol [Internet]. 2011 [cited 2018 Sep 4];86(5):1011-5. Available from: http://dx.doi.org/10.1590/ S0365-05962011000500024

4. World Health Organization. Leprosy elimination. Epidemiology: Epidemiological situation, burden 
and distribution [Internet]. 2018 [cited 2018 Sep 4]. Available from: http://www.who.int/lep/ epidemiology/en/

5. World Health Organization. Weekly epidemiological record. Relevé épidémiologique hebdomadaire [Internet]. 2014 [cited 2017 June 20];89(36):389-400. Available from: http://www. who.int/wer/2014/wer8936.pdf?ua=1

6. GámezSD, Ferrer BMJ, Fonseca MN. Caracterización de los pacientes con enfermedad de Hansen en un área de salud del municipio de Songo-La Maya. MEDISAN [Internet]. 2013 ago [cited 2016 June 17];17(8):3018-22. Available from: http://scielo. sld.cu/scielo.php?script=sci_arttext\&pid=S102930192013000800005\&lng=es

7. Brasil. Ministério da Saúde. Situação epidemiológica da hanseníase - dados [Internet]. Brasília; 2014 [cited 2016 Feb 20]. Available from: http://portalsaude.saude.gov.br/ index.php/o-ministerio/principal/leia-mais-oministerio/705-secretaria-svs/vigilancia-de-a-a-z/ hanseniase/11298-situacao-epidemiologica-dados

8. Brasil. Ministério da Saúde. Acompanhamento dos dados de hanseníase - Alagoas [Internet]. Brasília; 2015 [cited 2017 Mar 10]. Available from: http:// tabnet.datasus.gov.br/cgi/tabcgi.exe?sinannet/ hanseniase/cnv/hanswal.def

9. Alagoas. Indicadores e dados selecionados de saúde em Alagoas [Internet]. Maceió; 2016 [cited 2017 June 25]. Available from: http://www.riolargo. al.gov.br/

10. Brasil. Ministério da Saúde. Guia de vigilância em Saúde [Internet]. Brasília; 2014 [cited 2017 Mar 15]. Available from: http://portalarquivos.saude.gov.br/ images/pdf/2014/novembro/27/guia-vigilanciasaude-linkado-27-11-14.pdf

11. Garcia DR, Ignotti E, Cortela DCB, Xavier DR, Barelli CSGAP. Análise espacial dos casos de hanseníase, com enfoque à área de risco, em uma unidade básica de saúde no município de Cáceres (MT). Cad saúde colet [Internet]. 2013 [cited 2016 Mar 4];21(2):168-72. Available from: http://dx.doi. org/10.1590/S1414-462X2013000200011

12. Rodrigues FF, Calou CGC, Leandro TA, Antezana FJ, Pinheiro AKB, Silva VM, et al. Conhecimento e prática dos enfermeiros sobre hanseníase: ações de controle e eliminação. Rev Bras Enferm [Internet]. 2015 [cited 2018 Sep 4];68(2):297-304. Available from: http://www.redalyc.org/articulo. oa?id=267040408016
13. Brasil. Ministério da Saúde. Hanseníase. Boletim epidemiológico [Internet]. 2018 [cited 2018 Sep 1];49(4):1-12. Available from: http:// portalarquivos2.saude.gov.br/images/pdf/2018/ janeiro/31/2018-004-Hanseniase publicacao.pdf

14. Romanholo HSB, Souza EA, Ramos Júnior AN, Kaiser ACGCB, Silva IO, Brito AL, et al. Surveillance of intradomiciliary contacts of leprosy cases: perspective of the client in a hyperendemic municipality. Rev Bras Enferm [Internet]. 2018 [cited 2018 Sep 1];71(1):163-9. Available from: http://dx.doi.org/10.1590/0034-7167-2016-0607

15. Souza EA, Boigny RN, Ferreira AF, Alencar CH, Oliveira MLW, Ramos Jr AN. Vulnerabilidade programática no controle da hanseníase: padrões na perspectiva de gênero no Estado da Bahia, Brasil. Cad Saúde Pública [Internet]. 2018 [cited 2018 Sep 1];34(1):e00196216. Available from: http://dx.doi.org/10.1590/0102-311x00196216

16. Marinho FD, Nardi SMT, Coutinho GC, Sime MM. Hanseníase em menores de 15 anos: uma revisão de bibliográfica. REFACS [Internet]. 2015 [cited 2017 Mar 15];3(2):95-105.Available from: http:// seer.uftm.edu.br/revistaeletronica/index.php/ refacs/article/view/1087/pdf_1

17. Táquez AE, Cerón CA, Chaparro MA, Sales AM, Nery JA, Miranda A, et al. Lepra en la infancia: desafío diagnóstico. Rev argent dermatol [Internet]. 2011 [cited 2017 Mar 15];92(4):2-10. Available from: http://www.scielo.org.ar/scielo.php?script=sci_ arttext\&pid=S1851300X2011000400002\&lng=es

18. Amin GA, Lucena BD, Pires CAA. Infiltration in a child's face due to borderline lepromatous leprosy. Rev Soc Bras Med Trop [Internet]. 2017 [cited 2018 Sep 1];50(5):743. Available from: http://dx.doi. org/10.1590/0037-8682-0376-2016

19. Freitas BHBM, Xavier DR, Cortela SCB, Ferreira SMB. Leprosy in individuals under the age of fifteen in priority cities, Mato Grosso, Brazil. Rev Bras Epidemiol [Internet]. 2018 [cited 2018 Sep 1];21:e180016. Available from: http://dx.doi. org/10.1590/1980-549720180016

20. Pinto ACVD, Silva GV, Wachholz PA, Masuda PY. Profile of leprosy in children under 15 years of age monitored in a Brazilian referral center (20042012). An Bras Dermatol [Internet]. 2017 [cited 2018 Sep 1];92(4):580-2. Available from: https://www. ncbi.nlm.nih.gov/pmc/articles/PMC5595619/pdf/ abd-92-04-0580.pdf 
Fernanda da Silva Goes, Clodis Maria Tavares, Jovânia Marques de Oliveira e Silva, Tâmyssa Simões dos Santos, Nataly Mayara Cavalcante Gomes, Karen da Silva Santos

21. Lopes VAS, Rangel EM. Hanseníase e vulnerabilidade social: uma análise do perfil socioeconômico de usuários em tratamento irregular. Saúde debate [Internet]. 2014 [cited 2018 Sep 1];38(103):817-29. Available from: http://www.scielo.br/pdf/sdeb/v38n103/01031104-sdeb-38-103-0817.pdf

22. Cunha MHC, Silvestre MPSA, Silva AR, Rosário DDS, Xavier MB. Fatores de risco em contatos intradomiciliares de pacientes com hanseníase utilizando variáveis clínicas, sociodemográficas e laboratoriais. Rev Pan-Amaz Saude [Internet]. 2017 [cited 2018 Sep 1];8(2):23-30. Available from: http:// dx.doi.org/10.5123/s2176-62232017000200003

23. Luna ICF, Moura LTR, Vieira MCA. Clinical epidemiological profile of leprosy in children under 15 years in the city of Juazeiro-BA. Rev Bras Promoc Saude [Internet]. 2013 [cited 2018 Sep 1];26(2):202-9. Available from: http://periodicos. unifor.br/RBPS/article/view/2906/pdf_1

Recebido: 30 de março de 2018 Aprovado: 23 de setembro de 2018

Publicado: 27 de novembro de 2018

A Revista Baiana de Enfermagem utiliza a Licença Creative Commons - Atribuição-NãoComercial 4.0 Internacional. https://creativecommons.org/licenses/by-nc/4.0/

Este artigo é de acesso aberto distribuído sob os termos da Licença Creative Commons (CC BY-NC). Esta licença permite que outros remixem, adaptem e criem a partir do seu trabalho para fins não comerciais. Embora os novos trabalhos tenham de lhe atribuir o devido crédito e não possam ser usados para fins comerciais, os usuários não têm de licenciar esses trabalhos derivados sob os mesmos termos. 